\title{
GLOBAL STABILITY OF A BIOLOGICAL MODEL WITH TIME DELAY
}

\author{
SUZANNE M. LENHART AND CURTIS C. TRAVIS
}

\begin{abstract}
This paper gives necessary and sufficient conditions for global stability of certain logistic delay differential equations for all values of the delay.
\end{abstract}

Biological models frequently lead to delay differential equations and to questions concerning the stability of equilibrium solutions of such models. The monographs by Cushing [6] and MacDonald [16] discuss a number of examples of such models from population dynamics, ecology, and physiology. Much of the work with such models has focused on delay equations which are reducible, through a change of variables, to systems of differential equations without delays $[3,4,8,14,18,19]$.

Another line of research is concerned with conditions under which linear retarded functional differential equations of the form

$$
\dot{x}(t)=a x(t)+\sum_{i=1}^{L} b_{i} x\left(t-r_{i}\right), \quad r_{i} \geqslant 0,
$$

are asymptotically stable for all values of the delay $[1,3,5,7,9,11,12,14]$. We propose to combine these lines of research and examine global stability of the widely used logistic model of population dynamics,

$$
\begin{gathered}
\dot{x}(t)=x(t)\left(r+a x(t)+\sum_{i=1}^{L} b_{i} x\left(t-r_{i}\right)\right), \quad r_{i} \geqslant 0, \\
x(t)=\phi(t) \quad \text { for }-r \leqslant t \leqslant 0,
\end{gathered}
$$

where $r=\max \left\{r_{i}\right\}$.

We establish global stability (with respect to solutions with positive initial conditions) of the positive equilibrium $\bar{x}$ of equation (1). The hypothesis of our theorem guarantees that such an equilibrium exists. Koslesov [13] and Mazorov [15] point out that equation (1) can possess periodic solutions, while Beddington and May [2] point out that it need not do so. Hence the question of global stability of equation (1) is of interest.

THEOREM. Equation (1) is globally stable for initial conditions $\phi$ satisfying $\phi(0) \geqslant 0$ and for all $r_{i} \geqslant 0$ if and only if

$$
\sum_{i=1}^{L}\left|b_{i}\right| \leqslant-a
$$

Received by the editors August 23, 1984.

1980 Mathematics Subject Classification. Primary 43D20.

Key words and phrases. Lotka-Volterra delay differential equation, global stability, Liapunov function.

(c) 1986 American Mathematical Society $0002-9939 / 86 \$ 1.00+\$ .25$ per page 
and

$$
\sum_{i=1}^{L} b_{i}<-a
$$

Proof. First we assume conditions (2) and (3) hold. Let $\bar{x}$ be a positive equilibrium for equation (1) and let $y=x-\bar{x}$. Then

$$
\frac{d y}{d t}=(\bar{x}+y(t))\left(a y(t)+\sum_{i=1}^{L} b_{i} y\left(t-r_{i}\right)\right) .
$$

For each nonzero $b_{i}$, let $d_{i}$ be a positive constant to be chosen later. Define the positive definite functional $V: C(-r, 0) \rightarrow \mathbf{R}$ by

$$
V(\phi)=\phi(0)-\bar{x} \ln \left(\frac{\phi(0)+\bar{x}}{\bar{x}}\right)+\sum_{i=1}^{L} d_{i} \int_{-r_{i}}^{0} \phi^{2}(\theta) d \theta .
$$

We will prove that $V$ is a Liapunov function for equation (1). Using notation from Hale [10],

$$
\begin{aligned}
\dot{V}(\phi) & =a \phi^{2}(0)+\sum_{i=1}^{L} b_{i} \phi\left(-r_{i}\right) \phi(0)+\phi^{2}(0) \sum_{i=1}^{L} d_{i}-\sum_{i=1}^{L} d_{i} \phi^{2}\left(-r_{i}\right) \\
& \leqslant a \phi^{2}(0)+\sum_{i=1}^{L}\left|b_{i}\left\|\phi\left(-r_{i}\right)\right\| \phi(0)\right|+\phi^{2}(0) \sum_{i=1}^{L} d_{i}-\sum_{i=1}^{L} d_{i} \phi^{2}\left(-r_{i}\right) \\
& =-z^{T} A z
\end{aligned}
$$

where $z^{T}=\left(|\phi(0)|,\left|\phi\left(-r_{1}\right)\right|,\left|\phi\left(-r_{2}\right)\right|, \ldots,\left|\phi\left(-r_{L}\right)\right|\right)$ and $A$ is the $(L+1)$ square matrix

$$
A=\left[\begin{array}{ccccc}
-a-\sum_{i=1}^{L} d_{i} & -\left|b_{1}\right| / 2 & -\left|b_{2}\right| / 2 & \cdots & -\left|b_{L}\right| / 2 \\
-\left|b_{1}\right| / 2 & d_{1} & 0 & \cdots & 0 \\
-\left|b_{2}\right| / 2 & 0 & d_{2} & \cdots & 0 \\
\vdots & \vdots & \vdots & & \vdots \\
-\left|b_{L}\right| / 2 & 0 & 0 & \cdots & d_{L}
\end{array}\right]
$$

We will have

$$
-z^{T} A z \leqslant-\lambda_{\min }|\phi(0)|^{2}<0,
$$

where $\lambda_{\min }$ is the smallest eigenvalue of $A$, if matrix $A$ is positive definite. Since the off-diagonal elements are nonpositive, $A$ is positive definite if and only if all the principal minors of $A$ are positive [17]. This condition implies that the constants $d_{1}, \ldots, d_{L}$ must be chosen so that

$$
\sum_{i=1}^{L} \frac{b_{i}^{2}}{4 d_{i}}+\sum_{i=1}^{L} d_{i}<-a .
$$


First, consider the case where

$$
\sum_{i=1}^{L}\left|b_{i}\right|<-a,
$$

and choose $d_{i}=\left|b_{i}\right| / 2$. Inequality (4) holds for this choice of $d_{i}$ 's. Thus, equation (1) is globally stable when (5) holds.

Consider the case when

$$
\sum_{i=1}^{L}\left|b_{i}\right|=-a \text { and } \sum_{i=1}^{L} b_{i}<-a .
$$

These two conditions imply that some $b_{i}$ is negative. For the same choice of $d_{i}$, note that

$$
a=-\sum_{i=1}^{L}\left|b_{i}\right|=-2 \sum_{i=1}^{L} d_{i}
$$

and that we can now only show that $\dot{V}(\phi) \leqslant 0$.

To circumvent this problem, consider the set $S=\{\phi: \dot{V}(\phi)=0\}$.

Using (7) and completing the square,

$$
\begin{aligned}
\dot{V}(\phi)= & -\left[\left(-a-\sum_{i=1}^{L} d_{i}\right) \phi^{2}(0)+\sum_{i=1}^{L}\left(d_{i} \phi^{2}\left(-r_{i}\right)-b_{i} \phi(0) \phi\left(-r_{i}\right)\right)\right] \\
= & -\left[\left(-a-\sum_{i=1}^{L} d_{i}-\sum_{i=1}^{L} \frac{b_{i}^{2}}{4 d_{i}}\right) \phi^{2}(0)\right. \\
& \left.+\sum_{i=1}^{L} d_{i}\left(\phi^{2}\left(-r_{i}\right)-\frac{b_{i}}{d_{i}} \phi(0) \phi\left(r_{i}\right)+\left(\frac{b_{i}}{2 d_{i}}\right)^{2} \phi^{2}(0)\right)\right] \\
= & -\left[\left(-a-\sum_{i=1}^{L}\left(d_{i}+\frac{b_{i}^{2}}{4 d_{i}}\right)\right) \phi^{2}(0)+\sum_{i=1}^{L} d_{i}\left(\phi\left(-r_{i}\right)-\frac{b_{i}}{2 d_{i}} \phi(0)\right)^{2}\right]=0 .
\end{aligned}
$$

Since $-a-\sum_{i=1}^{L}\left(d_{i}+b_{i}^{2} / 4 d_{i}\right)=0$,

$$
S=\left\{\phi: \phi\left(-r_{i}\right)=\left(b_{i} / 2 d_{i}\right) \phi(0) \text { for all } i=1, \ldots, L\right\} .
$$

Now determine the largest set $M$ in $S$ which is invariant with respect to equation (1). $M$ must be the set of initial values of solutions satisfying

$$
y\left(t-r_{i}\right)=\left(b_{i} / 2 d_{i}\right) y(t) \text { for all } i=1, \ldots, L .
$$

This implies

$$
\begin{aligned}
\frac{d y(t)}{d t} & =(\bar{x}+y(t))\left(a y(t)+\sum_{i=1}^{L} b_{i} y\left(t-r_{i}\right)\right) \\
& =(\bar{x}+y(t))\left(a+\sum_{i=1}^{L} \frac{b_{i}^{2}}{2 d_{i}}\right) y(t)=0 \text { by (7). }
\end{aligned}
$$

Thus $y(t)=c$, a constant. But (8) gives $c=\left(b_{i} / 2 d_{i}\right) c$ for all $i=1, \ldots, L$. Since some $b_{i}$ is negative, $b_{i} / 2 d_{i} \neq 1$ for some $i$, and we must have $c=0$. By Theorem 3.1 of Hale [10], equation (1) is globally asymptotically stable. 
To prove the converse, let $y=x-\bar{x}$ and linearize the transformed equation about $y=0$ to obtain

$$
\frac{d y}{d t}=\bar{x}\left(a y(t)+\sum_{i=1}^{L} b_{i} y\left(t-r_{i}\right)\right) .
$$

From Corollary 1.2 of Lenhart and Travis [14], equation (9) is stable if and only if conditions (2) and (3) hold. Since global stability of the nonlinear equation (1) implies stability of the linearized equation (9), conditions (2) and (3) must hold.

\section{REFERENCES}

1. H. R. Bailey and M. Z. Williams, Some results on the differential difference equation $x^{\prime}(t)=$ $\sum_{i=0}^{n} A_{i} x\left(t-T_{i}\right)$, J. Math. Anal. Appl. 15 (1966), 569-587.

2. J. R. Beddington and R. M. May, Time delays are not necessarily destabilizing, Math. Biosci. 27 (1975), 109-117.

3. S. N. Busenberg and C. C. Travis, On the use of reducible-functional differential equations in biological models, J. Math. Anal. Appl. 89 (1982), 46-66.

4. D. S. Cohen, E. Coutsias, and J. Neu, Stable oscillations in single species growth models with hereditary effects, Math. Biosci. 44 (1979), 255-267.

5. K. L. Cooke and J. M. Ferreira, Stability conditions for linear retarded functional differential equations, preprint.

6. J. M. Cushing, Integrodifferential equations and delay models in population dynamics, Lecture Notes in Biomathematics, no. 20, Springer-Verlag, Berlin, 1977.

7. R. Datko, A procedure for determination of the exponential stability of certain differential-difference equations, Quart. Appl. Math. 36 (1978), 279-292.

8. D. M. Fargue, Reducibilité des sytèmes hereditaires a des systèmes dynamiques, C. R. Acad. Sci. Paris, Sér. B. 277 (1973), 471-473.

9. J. K. Hale, Sufficient conditions for stability and instability of autonomous functional-differential equations, J. Differential Equations 1 (1965), 452-482.

10. __ Theory of functional differential equations, Springer-Verlag, New York, 1977.

11. J. K. Hale, E. F. Infante, and F. P. Tsen, Stability in linear delay equations, Lefschetz Center for Dynamical Systems, Brown University Report \# 82-23.

12. N. D. Hayes, Roots of the transcendental equation associated with a certain differential-difference equation, J. London Math. Soc. (2) 25 (1950), 226-232.

13. U. S. Koslesov, Properties of solutions of a class of equations with lag which describe the dynamics of change in the population of a species with the age structure taken into account, Math. USSR-Sb. 45 (1983), 91-100.

14. S. M. Lenhart and C. C. Travis, Stability of functional partial differential equations, J. Differential Equations (to appear).

15. A. Mazarov, On the differential-difference growth equation, Search 4 (1973), 199-201.

16. N. McDonald, Time lags in biological models, Lecture Notes in Biomathematics, no. 27, SpringerVerlag, Berlin, 1978.

17. R. V. Plemmons, M-matrix characterizations. I: Nonsingular M-matrices, Linear Algebra Appl. 18 (1977), 175-188.

18. W. M. Post and C. C. Travis, Global stability in ecological models with continuous time delays, Integral and Functional Differential Equations (T. Herdman, H. Stech, and S. Rankin, Eds.), Dekker, New York, 1981, pp. 241-249.

19. A. Worz-Busekros, Global stability in ecological systems with continuous time delays, SIAM J. Appl. Math. 35 (1978), 123-134.

Department of Mathematics, University of Tennessee, KNoXville, Tennessee 37996-1300

Health and Safety Research Division, Oak Ridge National Laboratory, Oak Ridge, TennesSEE 37831 